APHASIOLOGY, 1998 , vol. 12 , No. $7 / 8,547-560$

\title{
Conversational discourse analysis: appropriate and useful sample sizes
}

\author{
LARRY BOLES and TODD BOMBARD \\ University of Hawai'i, Honolulu, Hawaii, USA
}

\begin{abstract}
The time required to transcribe and analyse lengthy conversation puts conversational discourse analysis (CDA) out of reach for most practising clinicians. However, standards have not been established for appropriate conversation sample size. Data are presented supporting the use of conversation samples of 5-10 minutes when studying conversation repair, speaking rate, and utterance length. Ten minute samples adequately represented 'parent' conversations from which they were derived when measuring conversation repair for six of eight cases. For measuring speaking efficiency (length of utterance and speaking rate) 5 minutes was adequate for all eight cases. For variables occurring once per minute, 10 minute samples were adequate, and for variables occurring three times per minute, 5 minute samples were adequate.
\end{abstract}

\section{Introduction}

Conversation has been used to study several populations and communicative contexts recently, including children with specific language impairment (Fujiki $e t$ al. 1990, Tomasello et al. 1990), psychotherapy interactions (Weingarten 1992), the study of gossip (Besnier 1989), traumatic brain injury (Damico and Housewright 1992, Damico and Schweitzer 1991) and aphasia (Boles 1996, 1997, Ferguson 1994, Perkins 1995). Conversational discourse analysis (CDA) has not enjoyed widespread clinical use, possibly because of the time required for transcription and analysis. Sampling of conversational discourse is one method for circumventing the laborious task of transcribing lengthy conversations. However, the issue of sample duration becomes problematic when the variables examined are unevenly dispersed. For example, when a variable occurs once every 20 minutes, a 5 minute sample is unlikely to estimate it adequately. Further, if the variable occurs eight times in 1 minute and not at all for 10 minutes, a 5 minute sample is unsatisfactory.

In a study involving analysis of clinician-patient interactions, Brookshire et al. (1978) concluded that any sampling procedure should sample events distributed throughout sessions. For example, a series of 1 minute samples taken every 5 minutes resulted in only $3 \%$ error for 39 categories. Brookshire and Nicholas (1994) concluded that short connected speech samples were highly unstable from test to test. The purpose of the current study is to present data supporting the use of conversation discourse samples of 5-10 minutes when studying conversation repair, speaking rate, and utterance length. The current study is one approach to quantifying the degree to which events are evenly distributed, as expressed by Brookshire et al. (1978) and Brookshire and Nicholas (1994).

It is important to note that the method of CDA is quite different from the method Address correspondence to: Larry Boles Ph.D., Assistant Professor, University of Hawai'i, 1410 Lower Campus Rd, Honolulu, HI 96822, Hawaii, USA. 


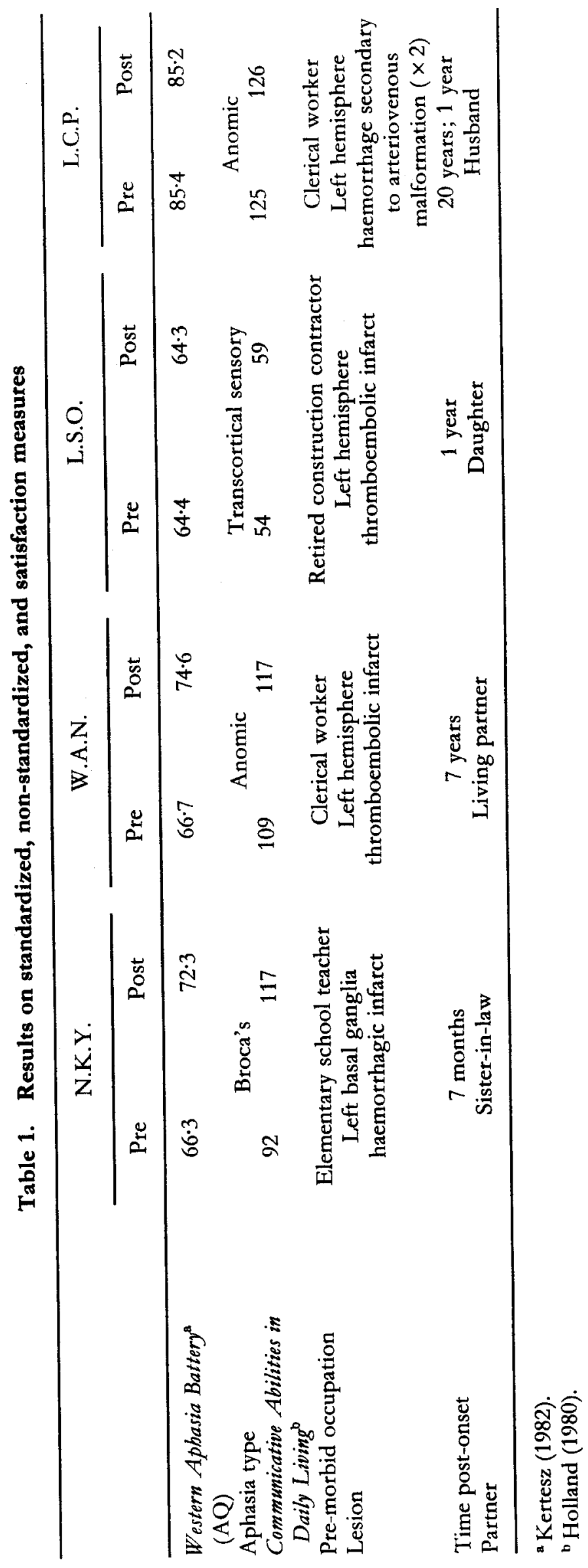


Table 2. Measures of verbal output

Words

Utterances

Self-repair

Other-repair

1. Cue

2. Model

3. Reflection

4. Request for clarification

5. Undifferentiated request for clarification
Total words spoken by participant during the sample

Excluded were gestures and other non-verbal communicative efforts

Total utterances spoken by participant during the sample.

These were defined according to guidelines by Kennedy $e t$ al. (1994) as follows:

1. a period of silence by the speaker signalling the relinquishment of the turn;

2. an intonational change by the speaker signalling the relinquishment of the turn;

3. the taking of a turn, or interruption, in the absence of a signal by the speaker;

4. the grammatical completion of an ideational unit or strings of ideational units; or

5. one-word utterances such as 'yes', 'oh', 'uh-huh', if occurring with characteristics 1 or 2

Including self-correction, repeating oneself, paraphrasing oneself, etc.

Any 'hint' given where it appears the speaker is aware of the target utterance and is attempting to induce that target Often follows a cue, where the speaker simply speaks the target utterance for the other participant

Rephrasing or paraphrasing what the other participant has said. May be used specifically to induce a known target, and/or simply as a kind of 'bookmark' to keep the conversation flowing

A specific request for a repetition or rephrasing when the speaker did not understand the other participant's utterance Similar to request for clarification above, but often expressed as simply, 'what?', 'huh?', etc.

of conversation analysis (CA). First, the focus of CA is always on the case rather than on groups of individuals. Second, CA is designed to capture the most salient properties of conversation and requires the researcher to let the conversation 'reveal itself' (Psathas 1995). That is, the researcher does not determine a priori what variables are most salient in a conversation. In the present study, five variables were chosen for study from conversational discourse samples obtained in a clinical environment. The reader is referred to Simmons-Mackie and Damico (1996) for a $\mathrm{CA}$ analysis which demonstrates usefulness for the clinician.

\section{Methods}

\section{Participants}

Four dyads comprising an adult with aphasia and his or her significant other engaged in weekly conversations for use in previous research (Boles 1997). In that study, CDA was used to monitor the communicative progress of four individuals with aphasia. Subjects were consecutive admissions to a university clinic who had (i) a diagnosis of aphasia; (ii) a conversational partner willing to participate; and (iii) no prior history of hearing, vision, psychiatric or other neurologic impairments. Demographic information appears in table 1. Eleven conversations 


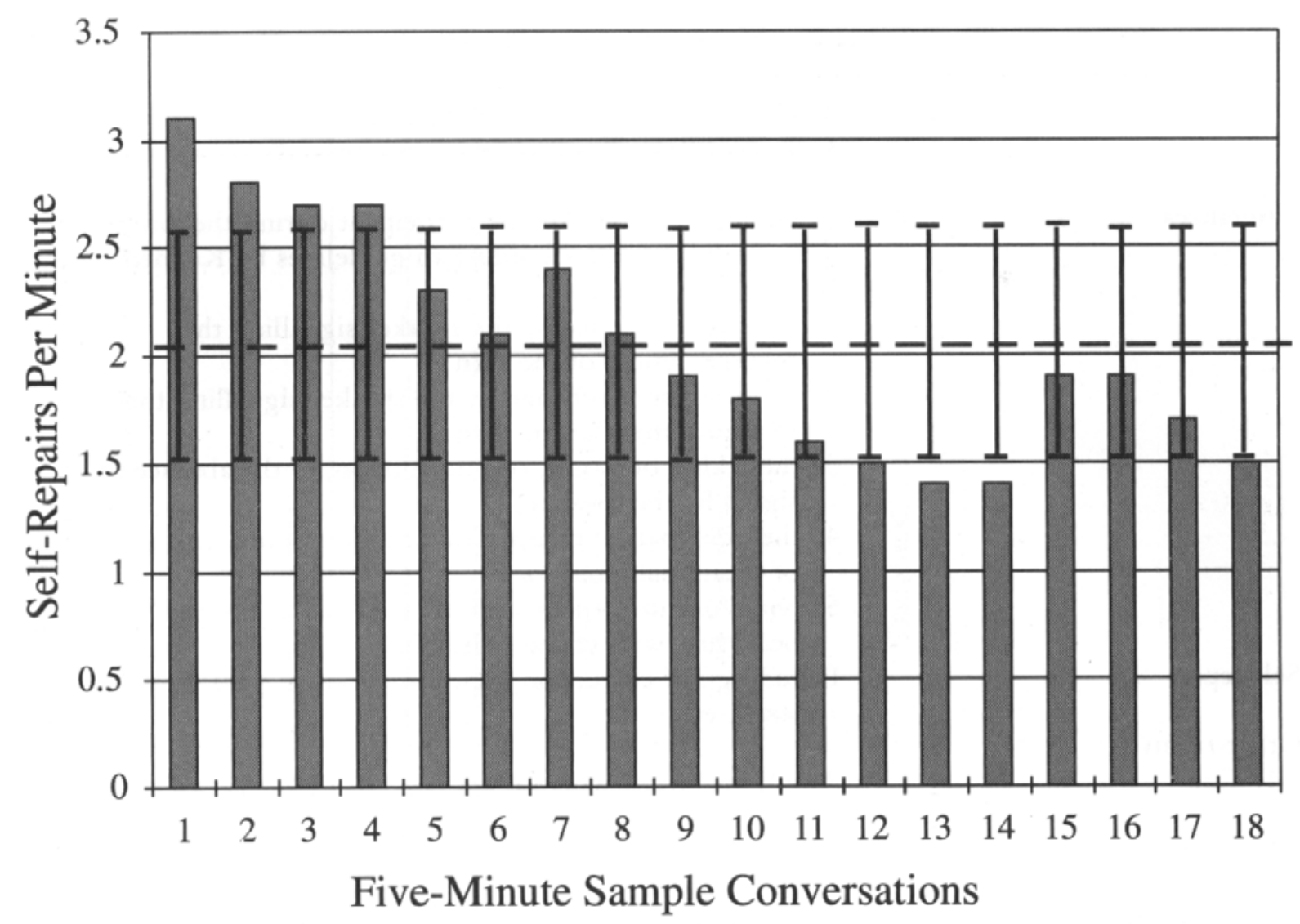

Figure 1. Eighteen conversations representing a parent conversation. The mean of the parent conversation $(2 \cdot 1)$ is represented by the dashed line. Coefficient of variation for these data is $0 \cdot 25$. Thus, the standard deviation of this distribution (represented by the error bars) is $25 \%$ of the mean for the parent conversation.

of 15-40 minutes were obtained per dyad - the first three on consecutive days and the following eight at weekly intervals. These conversations were elicited in the clinic prior to the first therapy session each week. Participants were simply instructed to talk about anything they wished. Paper and pencils were present, but no extraneous stimuli were provided.

\section{Conversational discourse analysis}

Conversations were videotaped and transcribed orthographically. Measures of verbal efficiency (see table 2) comprised words per utterance, frequency of words, utterances, and conversation repair (Sacks et al. 1974). Conversation repair was defined as an attempt to modify one's own or the other person's utterance for the purpose of clarification. Repairs were further classified into self- and other-repair (table 2).

\section{Reliability}

A second judge transcribed and coded $20 \%$ of all conversational interaction. For this purpose, nine of the 44 conversations were re-transcribed and recoded by the investigator and by a trained graduate student. These nine conversations were chosen at random from the pool of conversations. Inter-judge reliability was established for transcribed words (13975/14558 agreements, 0.96), utterance 


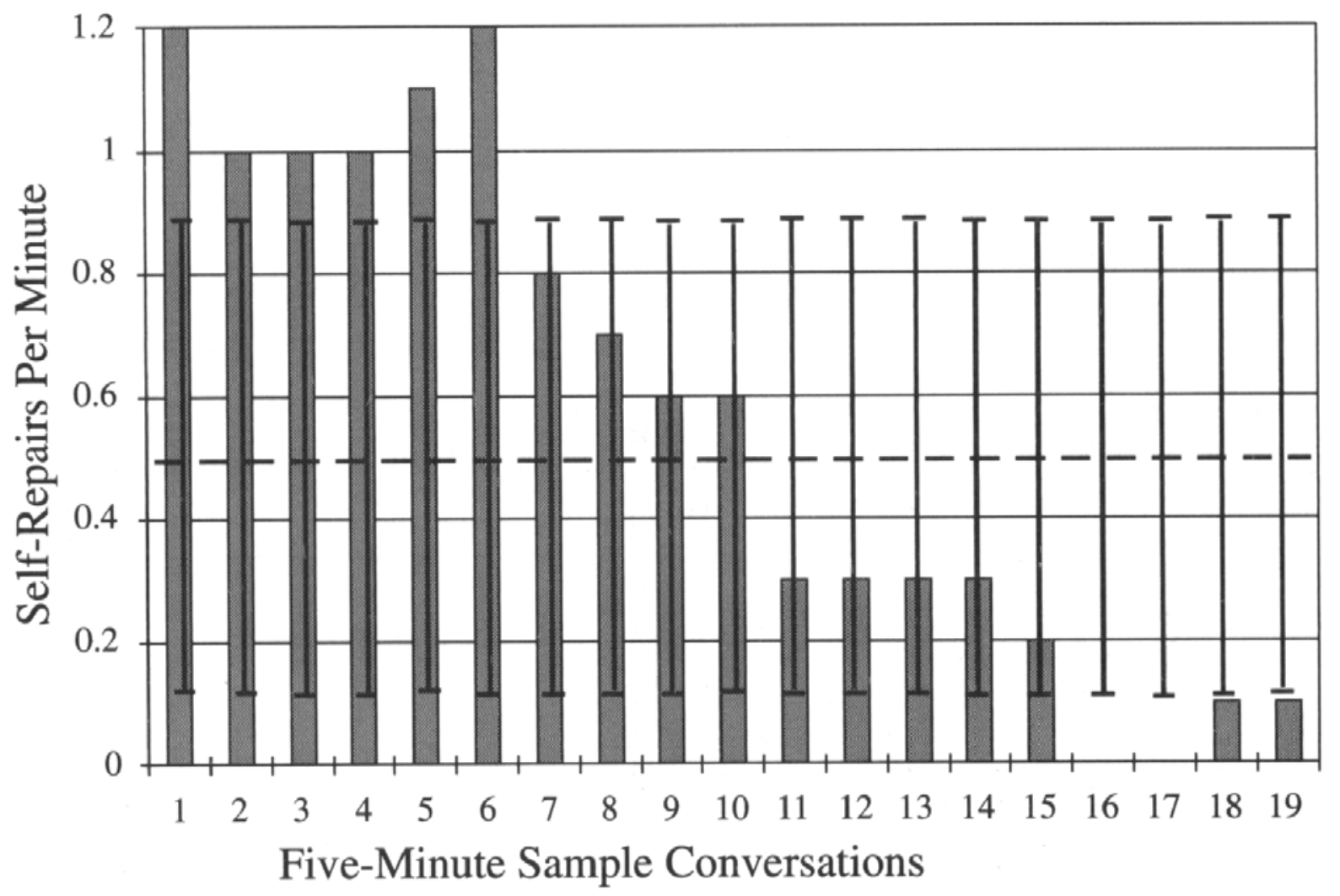

Figure 2. Nineteen conversations representing a parent conversation. The mean of the parent conversation $(0.50)$ is represented by the dashed line. Coefficient of variation for these data is $0 \cdot 74$. Thus, the standard deviation of this distribution (represented by the error bars) is $74 \%$ of the mean for the parent conversation.

boundaries (2705/2847 agreements, 0.95) and for conversation repair (972/1130 agreements, 0.86). Intra-judge reliability was established for transcribed words (14415/14558 agreements, 0.99), utterance boundaries (2829/2847 agreements, 0.99), and for coding of conversation repair (1074/1130 agreements, 0.95). Disagreements were resolved by consensus.

\section{Coefficient of variation}

The above 44 'parent' conversations ( 11 per dyad) were segmented into 5 and 10 minute samples for comparison. Thus, a 20 minute parent conversation yielded 16 consecutive 5 minute samples, with $4 / 5$ overlap in each 5 minute sample. For example, minutes $0-5,1-6,2-7 \ldots 15-20$. Similarly, 11 consecutive 10 minute samples were obtained from a 20 minute parent conversation. Means and standard deviations were calculated for dependent variables in the samples.

The coefficient of variation (CV) is a measure of relative dispersion, defined as the standard deviation of the sampling distribution divided by the population mean (Arney 1990). The CV controls for the magnitude of case values. In the present study, for example, the number of words per utterance is vastly different for the person with Broca's aphasia compared to non-aphasic participants. A unitless number, the CV quantifies the degree to which, in this case, sample conversations adequately represent parent conversations.

The current authors considered the mean of the parent conversation to represent a population mean. Thus, the calculation of the CV for self-repair was obtained by 
Table 3. Frequency of occurrence for parent conversations, dyad 1

\begin{tabular}{|c|c|c|c|c|c|c|}
\hline $\begin{array}{l}\text { Conversation } \\
\text { number }\end{array}$ & Minutes & Self-repairs & Other-repairs & $\begin{array}{l}\text { Words per } \\
\text { utterance }\end{array}$ & $\begin{array}{l}\text { Words per } \\
\text { conversation }\end{array}$ & $\begin{array}{l}\text { Utterances per } \\
\text { conversation }\end{array}$ \\
\hline \multicolumn{7}{|l|}{ N.K.Y. } \\
\hline 1 & 40 & $21(0.53)$ & $2(0 \cdot 05)$ & $1 \cdot 1$ & $169(4 \cdot 23)$ & $160(4 \cdot 00)$ \\
\hline 2 & 40 & $20(0 \cdot 50)$ & $2(0.05)$ & $1 \cdot 1$ & $169(4 \cdot 23)$ & $160(4 \cdot 00)$ \\
\hline 3 & 40 & $7(0 \cdot 18)$ & $3(0.08)$ & $1 \cdot 2$ & $211(5 \cdot 28)$ & $181(4 \cdot 53)$ \\
\hline 4 & 27 & $11(0 \cdot 41)$ & $1(0.04)$ & $1 \cdot 4$ & $166(6 \cdot 15)$ & $120(4 \cdot 44)$ \\
\hline 5 & 36 & $18(0.50)$ & $0(0)$ & $1 \cdot 5$ & $181(5.03)$ & $118(3 \cdot 28)$ \\
\hline 6 & 28 & $16(0.57)$ & $1(0 \cdot 04)$ & $1 \cdot 4$ & $206(7 \cdot 36)$ & $146(5 \cdot 21)$ \\
\hline 7 & 27 & $56(2 \cdot 07)$ & $0(0)$ & $2 \cdot 1$ & $367(13.59)$ & $179(6.63)$ \\
\hline 8 & 28 & $35(1 \cdot 25)$ & $4(0 \cdot 14)$ & $1 \cdot 7$ & $279(9 \cdot 96)$ & $163(5 \cdot 82)$ \\
\hline 9 & 24 & $44(1.83)$ & $0(0)$ & $1 \cdot 7$ & $231(9 \cdot 63)$ & $134(5 \cdot 58)$ \\
\hline 10 & 25 & $39(1.56)$ & $2(0 \cdot 08)$ & $1 \cdot 8$ & $266(10.64)$ & $146(5 \cdot 84)$ \\
\hline 11 & 19 & $23(1 \cdot 21)$ & $1(0.05)$ & $1 \cdot 6$ & $185(9 \cdot 74)$ & $114(6 \cdot 00)$ \\
\hline \multicolumn{7}{|c|}{$\begin{array}{l}11 \quad 19 \quad 23(1 \cdot 21) \\
\text { T.A.N. (conversational partner) }\end{array}$} \\
\hline 1 & 40 & $52(1 \cdot 30)$ & $110(2 \cdot 75)$ & $7 \cdot 4$ & $2338(58 \cdot 45)$ & $317(7 \cdot 93)$ \\
\hline 2 & 40 & $32(0.80)$ & $159(3.98)$ & $6 \cdot 5$ & $1739(43 \cdot 48)$ & $269(6 \cdot 73)$ \\
\hline 3 & 40 & $36(0.90)$ & $123(3.08)$ & $7 \cdot 3$ & $2226(55 \cdot 65)$ & $305(7 \cdot 63)$ \\
\hline 4 & 27 & $36(1 \cdot 33)$ & $72(2.67)$ & $8 \cdot 8$ & $1910(70 \cdot 74)$ & $216(8 \cdot 00)$ \\
\hline 5 & 36 & $52(1.44)$ & $56(1.56)$ & $9 \cdot 8$ & $2284(63 \cdot 44)$ & $233(6 \cdot 47)$ \\
\hline 6 & 28 & $22(0.79)$ & $88(3.14)$ & $7 \cdot 1$ & $1617(57 \cdot 75)$ & $229(8 \cdot 18)$ \\
\hline 7 & 27 & $10(0 \cdot 37)$ & $120(4 \cdot 44)$ & $4 \cdot 1$ & $806(29.85)$ & $197(7 \cdot 30)$ \\
\hline 8 & 28 & $31(1 \cdot 11)$ & $80(2 \cdot 86)$ & $6 \cdot 1$ & $1444(51.57)$ & $238(8.50)$ \\
\hline 9 & 24 & $10(0 \cdot 42)$ & $63(2.63)$ & $5 \cdot 4$ & $808(33.67)$ & $150(6 \cdot 25)$ \\
\hline 10 & 25 & $9(0.36)$ & $106(3.44)$ & $5 \cdot 3$ & $890(35.60)$ & $168(6 \cdot 72)$ \\
\hline 11 & 19 & $24(1 \cdot 26)$ & $61(3.21)$ & $6 \cdot 2$ & $904(47 \cdot 58)$ & $146(7 \cdot 68)$ \\
\hline
\end{tabular}

Upper half of table contains data for N.K.Y., while the lower half contains data pertaining to T.A.N., the non-aphasic communication partner. Values outside parentheses represent occurrences per parent conversation. Values inside parentheses represent values per minute.

dividing the standard deviation for self-repair for the 10 minute segments by the mean number of self-repairs per minute for the parent conversation. This was computed for each of the eleven parent conversations for each member of the dyad, then repeated for the 5 minute segments.

When the $\mathrm{CV}$ is above 0.50 , it is advisable to abandon it as a measure of dispersion (McCormick 1941). Although this 0.50 figure is somewhat arbitrary, a graphic illustration should clarify. Figure 1 represents self-repair scores. The apparent low degree of variability in these 5 minute samples is reflected in the $\mathrm{CV}$ value of 0.25 . A value of 0.25 indicates that the standard deviation for that distribution is $25 \%$ as large as the mean for that distribution (see error bars in figures 1 and 2). Figure 2, derived from a different parent conversation, shows a similar scenario, with the variability reflected in the $C V$ of $0 \cdot 74$. Thus a 5 minute sample adequately represents the parent conversation for the data in figure 1, but not for the parent conversation in figure 2 .

\section{Results}

For clarification, tables 3-6 represent the values for each of the five variables in the parent conversations for dyads $1-4$, respectively. The CVs in tables 7-10 are based on 5 and 10 minute segments of the parent conversations represented in tables 3-6. 
Table 4. Frequency of occurrence for parent conversations, dyad 2

\begin{tabular}{|c|c|c|c|c|c|c|}
\hline $\begin{array}{l}\text { Conversation } \\
\text { number }\end{array}$ & Minutes & Self-repairs & Other-repairs & $\begin{array}{l}\text { Words per } \\
\text { utterance }\end{array}$ & $\begin{array}{l}\text { Words per } \\
\text { conversation }\end{array}$ & $\begin{array}{l}\text { Utterances per } \\
\text { conversation }\end{array}$ \\
\hline \multicolumn{7}{|l|}{ W.A.N. } \\
\hline 1 & 27 & $5(0 \cdot 19)$ & $16(0.59)$ & $3 \cdot 8$ & $661(24 \cdot 48)$ & $172(6 \cdot 37)$ \\
\hline 2 & 23 & $32(1.39)$ & $2(0.09)$ & $5 \cdot 5$ & $547(23 \cdot 78)$ & $100(4 \cdot 35)$ \\
\hline 3 & 26 & $20(0 \cdot 77)$ & $10(0 \cdot 39)$ & $4 \cdot 7$ & $424(16 \cdot 31)$ & $90(3.46)$ \\
\hline 4 & 24 & $30(1.25)$ & $9(0.38)$ & 3.9 & $456(19 \cdot 00)$ & $118(4.92)$ \\
\hline 5 & 19 & $17(0 \cdot 90)$ & $9(0.47)$ & $4 \cdot 0$ & $340(17.89)$ & $86(4.53)$ \\
\hline 6 & 18 & $14(0 \cdot 78)$ & $7(0.33)$ & $4 \cdot 0$ & $261(14.50)$ & $65(3.61)$ \\
\hline 7 & 18 & $22(1 \cdot 22)$ & $7(0 \cdot 39)$ & $4 \cdot 3$ & $408(22 \cdot 67)$ & $96(5.33)$ \\
\hline 8 & 17 & $14(0.82)$ & $8(0.47)$ & $4 \cdot 4$ & $297(17 \cdot 47)$ & $68(4 \cdot 00)$ \\
\hline 9 & 18 & $20(1 \cdot 11)$ & $8(0.44)$ & $6 \cdot 4$ & $403(22 \cdot 39)$ & $63(3.50)$ \\
\hline 10 & 23 & $11(0 \cdot 48)$ & $7(0.30)$ & $5 \cdot 7$ & $311(13 \cdot 52)$ & $55(2 \cdot 39)$ \\
\hline 11 & 21 & $24(1 \cdot 14)$ & $6(0 \cdot 29)$ & $4 \cdot 0$ & $497(23.67)$ & $124(5 \cdot 91)$ \\
\hline \multicolumn{7}{|c|}{ R.I.C. (conversational partner) } \\
\hline 1 & 27 & $27(1 \cdot 00)$ & $87(3 \cdot 22)$ & $8 \cdot 4$ & $1961(71 \cdot 26)$ & $233(8.56)$ \\
\hline 2 & 23 & $68(2.96)$ & $17(0 \cdot 74)$ & $11 \cdot 0$ & $1437(62 \cdot 48)$ & $131(5 \cdot 70)$ \\
\hline 3 & 26 & $65(2 \cdot 50)$ & $6(0 \cdot 23)$ & $11 \cdot 8$ & $1724(66 \cdot 31)$ & $146(5 \cdot 62)$ \\
\hline 4 & 24 & $74(3.08)$ & $41(1 \cdot 71)$ & $10 \cdot 0$ & $1658(69 \cdot 08)$ & $166(6.92)$ \\
\hline 5 & 19 & $44(2 \cdot 32)$ & $37(1.95)$ & $10 \cdot 0$ & $1133(59 \cdot 63)$ & $113(5 \cdot 95)$ \\
\hline 6 & 18 & $70(3.89)$ & $20(1 \cdot 11)$ & $12 \cdot 0$ & $1378(76 \cdot 56)$ & $115(6 \cdot 39)$ \\
\hline 7 & 18 & $53(2.94)$ & $39(2 \cdot 17)$ & $10 \cdot 3$ & $1358(75 \cdot 44)$ & $132(7 \cdot 33)$ \\
\hline 8 & 17 & $45(2 \cdot 65)$ & $11(0.65)$ & $11 \cdot 6$ & $1206(70 \cdot 94)$ & $104(6 \cdot 12)$ \\
\hline 9 & 18 & $70(3.89)$ & $4(0 \cdot 22)$ & $12 \cdot 3$ & $1360(75 \cdot 56)$ & $111(6 \cdot 17)$ \\
\hline 10 & 23 & $41(1 \cdot 78)$ & $18(0.78)$ & $10 \cdot 1$ & $1237(53 \cdot 78)$ & $122(5 \cdot 30)$ \\
\hline 11 & 21 & $32(1.52)$ & $29(1.38)$ & $8 \cdot 7$ & $1157(55 \cdot 10)$ & $133(6.33)$ \\
\hline
\end{tabular}

Upper half of table contains data for W.A.N., while the lower half contains data pertaining to R.I.C., the non-aphasic communication partner. Values outside parentheses represent occurrences per parent conversation. Values inside parentheses represent values per minute.

The authors arbitrarily decided that 'adequate representativeness' would be met when 8 of 11 sample conversations achieved a 0.50 cut-off point for the CV.

Dyad 1 (tables 3, 7 )

\section{Self-repair}

Self-repair was distributed such that a 10 minute sample adequately estimated that information for T.A.N., but not for N.K.Y. Five minute samples were not adequate.

\section{Other-repair}

Ten minute samples did not adequately represent parent conversations for otherrepair for N. K. Y. in any conversation. It should be noted that her frequency of other-repair was rarely more than twice per parent conversation (see table 4). For T.A.N., 10 minute samples adequately represented parent conversations. Five minute samples did not adequately represent parent conversations for N.K.Y. or T.A.N. 
Table 5. Frequency of occurrence for parent conversations, dyad 3

\begin{tabular}{|c|c|c|c|c|c|c|}
\hline $\begin{array}{l}\text { Conversation } \\
\text { number }\end{array}$ & Minutes & Self-repairs & Other-repairs & $\begin{array}{l}\text { Words per } \\
\text { utterance }\end{array}$ & $\begin{array}{l}\text { Words per } \\
\text { conversation }\end{array}$ & $\begin{array}{l}\text { Utterances per } \\
\text { conversation }\end{array}$ \\
\hline \multicolumn{7}{|l|}{ L.S.O. } \\
\hline 1 & 20 & $6(0 \cdot 30)$ & $64(3.20)$ & $2 \cdot 3$ & $485(24 \cdot 25)$ & $211(10 \cdot 55)$ \\
\hline 2 & 20 & $53(2 \cdot 65)$ & $44(2 \cdot 20)$ & $4 \cdot 4$ & $989(49 \cdot 45)$ & $224(11 \cdot 20)$ \\
\hline 3 & 18 & $14(0.78)$ & $21(1 \cdot 17)$ & $3 \cdot 1$ & $478(26.56)$ & $155(8 \cdot 61)$ \\
\hline 4 & 15 & $5(0.33)$ & $15(1.00)$ & $3 \cdot 5$ & $389(25.93)$ & $112(7 \cdot 47)$ \\
\hline 5 & 15 & $11(0 \cdot 73)$ & $21(1 \cdot 40)$ & $3 \cdot 8$ & $472(31 \cdot 47)$ & $123(8 \cdot 20)$ \\
\hline 6 & 15 & $19(1 \cdot 27)$ & $16(1.07)$ & $4 \cdot 5$ & $524(34.93)$ & $117(7 \cdot 80)$ \\
\hline 7 & 16 & $17(1 \cdot 06)$ & $13(0 \cdot 81)$ & $3 \cdot 4$ & $404(25 \cdot 25)$ & $120(7 \cdot 50)$ \\
\hline 8 & 15 & $66(4 \cdot 40)$ & $31(2 \cdot 07)$ & $4 \cdot 9$ & $1045(69 \cdot 67)$ & $214(14 \cdot 27)$ \\
\hline 9 & 15 & $48(3.20)$ & $9(0.60)$ & $4 \cdot 7$ & $773(51 \cdot 53)$ & $163(10 \cdot 87)$ \\
\hline 10 & 15 & $65(4 \cdot 33)$ & $7(0.47)$ & $5 \cdot 7$ & $874(58.27)$ & $153(10 \cdot 20)$ \\
\hline 11 & 15 & $52(3.47)$ & $19(1 \cdot 27)$ & $4 \cdot 0$ & $700(46 \cdot 67)$ & $175(11.67)$ \\
\hline \multicolumn{7}{|c|}{ S.Z.A. (conversational partner) } \\
\hline 1 & 20 & $104(5 \cdot 20)$ & $54(2 \cdot 70)$ & $5 \cdot 6$ & $1715(85 \cdot 75)$ & $304(15 \cdot 20)$ \\
\hline 2 & 20 & $86(4 \cdot 30)$ & $50(2.50)$ & $5 \cdot 8$ & $1450(72 \cdot 50)$ & $248(12 \cdot 40)$ \\
\hline 3 & 18 & $87(4 \cdot 83)$ & $51(2 \cdot 83)$ & $6 \cdot 7$ & $1468(81 \cdot 56)$ & $218(12 \cdot 11)$ \\
\hline 4 & 15 & $40(2 \cdot 67)$ & $48(3 \cdot 20)$ & $6 \cdot 4$ & $860(57 \cdot 33)$ & $134(8 \cdot 93)$ \\
\hline 5 & 15 & $30(2 \cdot 00)$ & $47(3 \cdot 13)$ & $5 \cdot 7$ & $810(54 \cdot 00)$ & $143(9 \cdot 53)$ \\
\hline 6 & 15 & $34(2 \cdot 27)$ & $42(2 \cdot 80)$ & $6 \cdot 0$ & $729(48 \cdot 60)$ & $122(8 \cdot 13)$ \\
\hline 7 & 16 & $34(2 \cdot 13)$ & $48(3.00)$ & $6 \cdot 8$ & $916(57 \cdot 25)$ & $135(8.44)$ \\
\hline 8 & 15 & $41(2 \cdot 73)$ & $42(2 \cdot 80)$ & $4 \cdot 7$ & $859(57 \cdot 27)$ & $223(12 \cdot 27)$ \\
\hline 9 & 15 & $14(0.93)$ & $77(5 \cdot 13)$ & $5 \cdot 1$ & $706(47.07)$ & $138(9 \cdot 20)$ \\
\hline 10 & 15 & $6(0.40)$ & $53(3.53)$ & $3 \cdot 8$ & $477(31 \cdot 80)$ & $124(8 \cdot 27)$ \\
\hline 11 & 15 & $42(2 \cdot 80)$ & $74(4.93)$ & $4 \cdot 4$ & $803(53.53)$ & $214(12 \cdot 20)$ \\
\hline
\end{tabular}

Upper half of table contains data for L.S.O., while the lower half contains data pertaining to S.Z.A., the non-aphasic communication partner. Values outside parentheses represent occurrences per parent conversation. Values inside parentheses represent values per minute.

Measures of verbal efficiency

For verbal efficiency, both 10 and 5 minute samples adequately represented parent conversations.

Dyad $2($ tables 4,8$)$

Self-repair

Ten minute samples adequately represented parent conversations for self-repair for both participants. Five minute samples were not adequate.

\section{Other-repair}

Ten minute samples adequately represented parent conversations for other repair for W.A.N., but not for R.I.C. Five minute conversations were inadequate estimates of parent conversations.

\section{Measures of verbal efficiency}

Both 5 and 10 minute samples adequately represented parent conversations for both participants in dyad 2 . 
Table 6. Frequency of occurrence for parent conversations, dyad 4

\begin{tabular}{|c|c|c|c|c|c|c|}
\hline $\begin{array}{l}\text { Conversation } \\
\text { number }\end{array}$ & Minutes & Self-repairs & Other-repairs & $\begin{array}{l}\text { Words per } \\
\text { utterance }\end{array}$ & $\begin{array}{l}\text { Words per } \\
\text { conversation }\end{array}$ & $\begin{array}{l}\text { Utterances per } \\
\text { conversation }\end{array}$ \\
\hline \multicolumn{7}{|c|}{$536(35 \cdot 73)$} \\
\hline 1 & 15 & $44(2.93)$ & $5(0 \cdot 33)$ & $5 \cdot 5$ & $536(35.73)$ & $97(6.47)$ \\
\hline 2 & 15 & $31(2 \cdot 07)$ & $4(0.27)$ & $4 \cdot 7$ & $479(31.93)$ & $103(6 \cdot 87)$ \\
\hline 3 & 15 & $46(3.07)$ & $9(0.60)$ & $4 \cdot 2$ & $525(35.00)$ & $125(8 \cdot 33)$ \\
\hline 4 & 15 & $32(2 \cdot 13)$ & $8(0.53)$ & $4 \cdot 2$ & $410(27 \cdot 33)$ & $98(6.53)$ \\
\hline 5 & 15 & $34(2 \cdot 27)$ & $4(0 \cdot 27)$ & $6 \cdot 5$ & $521(34 \cdot 73)$ & $80(5 \cdot 33)$ \\
\hline 6 & 15 & $44(2.93)$ & $7(0 \cdot 47)$ & $3 \cdot 8$ & $477(31 \cdot 80)$ & $126(8 \cdot 40)$ \\
\hline 7 & 15 & $41(2.73)$ & $6(0.40)$ & $4 \cdot 6$ & $499(33.27)$ & $108(7 \cdot 20)$ \\
\hline 8 & 15 & $53(3.53)$ & $2(0 \cdot 13)$ & $4 \cdot 3$ & $478(31.87)$ & $111(7 \cdot 40)$ \\
\hline 9 & 15 & $31(2 \cdot 07)$ & $4(0.27)$ & $5 \cdot 6$ & $611(40.73)$ & $109(7 \cdot 27)$ \\
\hline 10 & 15 & $25(1.67)$ & $6(0 \cdot 40)$ & $5 \cdot 1$ & $471(31 \cdot 40)$ & $92(6.13)$ \\
\hline 11 & 15 & $18(1 \cdot 20)$ & $9(0 \cdot 60)$ & $5 \cdot 1$ & $505(33.67)$ & $99(6.60)$ \\
\hline \multicolumn{7}{|c|}{$\begin{array}{l}11 \quad 15 \quad 18(1 \cdot 20) \\
\text { C.H.S. (Conversational partner) }\end{array}$} \\
\hline 1 & 15 & $12(0 \cdot 80)$ & $18(1 \cdot 20)$ & $4 \cdot 7$ & $349(23 \cdot 27)$ & $75(5 \cdot 00)$ \\
\hline 2 & 15 & $16(1.07)$ & $32(2 \cdot 13)$ & $5 \cdot 3$ & $502(33.47)$ & $95(6.33)$ \\
\hline 3 & 15 & $20(1 \cdot 33)$ & $35(2.33)$ & $4 \cdot 7$ & $514(34 \cdot 27)$ & $110(7 \cdot 33)$ \\
\hline 4 & 15 & $23(1.53)$ & $24(1 \cdot 60)$ & $6 \cdot 1$ & $608(40.53)$ & $100(6 \cdot 67)$ \\
\hline 5 & 15 & $12(0 \cdot 80)$ & $20(1 \cdot 33)$ & $5 \cdot 3$ & $342(22 \cdot 80)$ & $65(4 \cdot 33)$ \\
\hline 6 & 15 & $40(2 \cdot 67)$ & $32(2 \cdot 13)$ & $4 \cdot 9$ & $700(46 \cdot 67)$ & $142(9 \cdot 47)$ \\
\hline 7 & 15 & $39(2 \cdot 60)$ & $24(1.60)$ & $5 \cdot 3$ & $640(42.67)$ & $120(8 \cdot 00)$ \\
\hline 8 & 15 & $34(2 \cdot 27)$ & $26(1.73)$ & $4 \cdot 5$ & $504(33.60)$ & $112(7 \cdot 47)$ \\
\hline 9 & 15 & $8(0.53)$ & $15(1.00)$ & 4.9 & $374(24.93)$ & $76(5.07)$ \\
\hline 10 & 15 & $7(0.47)$ & $22(1.47)$ & $6 \cdot 2$ & $506(33.73)$ & $81(5 \cdot 40)$ \\
\hline 11 & 15 & $18(1 \cdot 20)$ & $20(1.33)$ & 6.4 & $709(47 \cdot 27)$ & $110(7 \cdot 33)$ \\
\hline
\end{tabular}

Upper half of table contains data for L.C.P., while the lower half contains data pertaining to C.H.S., the non-aphasic communication partner. Values outside parentheses represent occurrences per parent conversation. Values inside parentheses represent values per minute.

Dyad 3 (tables 5, 9)

\section{Self-repair}

Ten minute samples adequately represented parent conversations for self-repair in all conversations for both participants. Five minute samples were adequate for S.Z.A.

\section{Otber-repair}

Ten minute samples adequately represented parent conversations for other-repair for both participants. Five minute samples were adequate for S.Z.A.

\section{Measures of verbal efficiency}

Both 5 and 10 minute samples adequately represented parent conversations for both participants in dyad 3 . 
Table 7. Coefficients of variation for dyad 1 (N.K.Y. and T.A.N.)

\begin{tabular}{|c|c|c|c|c|c|c|c|c|c|c|}
\hline \multirow{2}{*}{$\begin{array}{l}\text { Conversation } \\
\text { number }\end{array}$} & \multicolumn{2}{|c|}{ Self-repair/min } & \multicolumn{2}{|c|}{ Other-repair/min } & \multicolumn{2}{|c|}{ Words/utterance } & \multicolumn{2}{|c|}{ Words/min } & \multicolumn{2}{|c|}{ Utterances/min } \\
\hline & $10 \mathrm{~min}$ & $5 \mathrm{~min}$ & $10 \mathrm{~min}$ & $5 \mathrm{~min}$ & $10 \mathrm{~min}$ & $5 \mathrm{~min}$ & $10 \mathrm{~min}$ & $5 \mathrm{~min}$ & $10 \mathrm{~min}$ & $5 \mathrm{~min}$ \\
\hline \multicolumn{11}{|l|}{ N.K.Y. } \\
\hline $1(40)$ & 0.56 & 0.98 & $1 \cdot 01$ & 1.82 & 0.08 & 0.22 & $0 \cdot 19$ & 0.42 & $0 \cdot 15$ & 0.35 \\
\hline $2(40)$ & 0.66 & 0.85 & $1 \cdot 20$ & $2 \cdot 24$ & 0.23 & 0.34 & 0.26 & 0.46 & 0.28 & $0 \cdot 41$ \\
\hline $3(40)$ & $1 \cdot 29$ & $1 \cdot 01$ & $3 \cdot 12$ & $1 \cdot 95$ & 0.04 & $0 \cdot 10$ & 0.24 & 0.26 & 0.22 & 0.23 \\
\hline $4(27)$ & 0.93 & 1.52 & $1 \cdot 38$ & 2.09 & 0.06 & 0.25 & 0.36 & 0.47 & 0.36 & 0.48 \\
\hline $5(36)$ & $0-67$ & $1 \cdot 00$ & $\mathrm{~N} / \mathrm{A}$ & N/A & $0 \cdot 21$ & 0.27 & 0.47 & 0.57 & $0 \cdot 29$ & 0.39 \\
\hline $6(28)$ & 0.74 & 0.92 & 0.88 & 1.58 & $0 \cdot 14$ & 0.32 & 0.37 & 0.51 & $0 \cdot 37$ & 0.48 \\
\hline $7(27)$ & 0.25 & 0.39 & $\mathrm{~N} / \mathrm{A}$ & $N / A$ & 0.08 & 0.19 & 0.09 & 0.21 & 0.02 & 0.03 \\
\hline $8(28)$ & $0 \cdot 21$ & 0.60 & 0.63 & $1 \cdot 14$ & 0.13 & 0.18 & 0.18 & 0.31 & 0.09 & 0.24 \\
\hline $9(24)$ & 0.21 & 0.24 & N/A & $\mathrm{N} / \mathrm{A}$ & 0.07 & 0.20 & 0.24 & 0.16 & 0.26 & 0.21 \\
\hline $10(25)$ & 0.24 & 0.38 & 1.07 & $1 \cdot 28$ & 0.08 & $0 \cdot 13$ & 0.17 & 0.26 & $0 \cdot 14$ & 0.23 \\
\hline $11(19)$ & 0.29 & 0.60 & 2.00 & 1.93 & 0.29 & 0.14 & 0.34 & 0.34 & 0.37 & 0.29 \\
\hline \multicolumn{11}{|l|}{ T.A.N. } \\
\hline $1(40)$ & 0.69 & 0.57 & 0.41 & 0.40 & 0.12 & 0.16 & 0.13 & 0.23 & 0.26 & 0.28 \\
\hline $2(40)$ & 1.26 & $1 \cdot 58$ & 0.27 & 0.38 & 0.26 & 0.32 & 0.30 & 0.40 & $0 \cdot 16$ & 0.29 \\
\hline $3(40)$ & 0.25 & 0.55 & 0.12 & 0.25 & 0.11 & 0.18 & 0.14 & 0.31 & 0.08 & 0.22 \\
\hline $4(27)$ & 1.08 & 0.77 & 0.84 & 0.55 & 0.30 & 0.26 & $0 \cdot 14$ & 0.25 & 0.30 & 0.24 \\
\hline $5(36)$ & 0.33 & 0.50 & 0.44 & 0.63 & $0 \cdot 11$ & 0.16 & 0.14 & 0.24 & $0 \cdot 11$ & 0.17 \\
\hline $6(28)$ & 0.13 & 0.61 & 0.55 & 0.71 & 0.16 & 0.32 & 0.12 & 0.27 & $0 \cdot 17$ & 0.24 \\
\hline $7(27)$ & 0.48 & 0.82 & 0.09 & $0 \cdot 16$ & 0.17 & $0 \cdot 30$ & 0.20 & 0.34 & 0.03 & 0.06 \\
\hline $8(28)$ & 0.44 & 0.68 & 0.18 & 0.36 & 0.18 & 0.28 & 0.22 & $0 \cdot 31$ & 0.07 & 0.16 \\
\hline $9(24)$ & 0.43 & 0.67 & 0.32 & 0.40 & 0.09 & $0 \cdot 21$ & 0.34 & 0.30 & 0.31 & 0.27 \\
\hline $10(25)$ & 0.35 & 0.81 & $0 \cdot 18$ & 0.39 & $0 \cdot 17$ & 0.33 & 0.12 & 0.24 & 0.54 & 0.87 \\
\hline $11(19)$ & 0.49 & 0.92 & 0.35 & 0.51 & 0.03 & $0 \cdot 14$ & 0.04 & 0.34 & 0.85 & $1 \cdot 24$ \\
\hline
\end{tabular}

Values of 0.50 or less represent 'acceptable' representations of parent conversations. Values in parentheses in the first column represent the length in minutes of parent conversations. Upper half of table contains data for N.K.Y.; the lower half contains data pertaining to T.A.N., the non-aphasic communication partner. ' $N / A$ ' refers to zero occurrences of that variable.

$$
\text { Dyad } 4 \text { (tables 6, 10) }
$$

Self-repair

Ten minute samples adequately represented parent conversations for self-repair for both participants. Five minute samples were adequate for L.C.P., but not for C.H.S.

\section{Other-repair}

Ten minute samples adequately represented parent conversations for other-repair for both participants. Five minute samples were adequate for C.H.S., but not for L.C.P.

\section{Measures of verbal efficiency}

Both 5 and 10 minute samples adequately represented parent conversations for both participants in dyad 4 .

\section{Overall pattern}

A clear pattern noted from the findings of this study was an even dispersion of variables, given a minimum occurrence of that variable. That is, given a frequency of occurrence of once per minute, a 10 minute segment was adequate in nearly all cases, and with a frequency of three times per minute, a 5 minute segment was 
Table 8. Coefficients of variation for dyad 2 (W.A.N. and R.I.C.)

\begin{tabular}{|c|c|c|c|c|c|c|c|c|c|c|}
\hline \multirow{2}{*}{$\begin{array}{l}\text { Conversation } \\
\text { number }\end{array}$} & \multicolumn{2}{|c|}{ Self-repair/min } & \multicolumn{2}{|c|}{ Other-repair/min } & \multicolumn{2}{|c|}{ Words/utterance } & \multicolumn{2}{|c|}{ Words/min } & \multicolumn{2}{|c|}{ Utterances/min } \\
\hline & $10 \mathrm{~min}$ & $5 \mathrm{~min}$ & $10 \mathrm{~min}$ & $5 \mathrm{~min}$ & $10 \mathrm{~min}$ & $5 \mathrm{~min}$ & $10 \mathrm{~min}$ & $5 \mathrm{~min}$ & $10 \mathrm{~min}$ & $5 \mathrm{~min}$ \\
\hline \multicolumn{11}{|l|}{ W.A.N. } \\
\hline $1(27)$ & 0.27 & 0.73 & 0.41 & 0.72 & $0 \cdot 13$ & $0 \cdot 23$ & $0 \cdot 25$ & $0 \cdot 32$ & $0 \cdot 30$ & 0.41 \\
\hline $2(23)$ & 0.47 & $0 \cdot 67$ & $0 \cdot 01$ & $1 \cdot 18$ & 0.07 & $0 \cdot 14$ & $0 \cdot 14$ & 0.29 & $0 \cdot 16$ & $0 \cdot 30$ \\
\hline $3(26)$ & 0.27 & 0.53 & 0.25 & 0.78 & $0 \cdot 17$ & 0.33 & $0 \cdot 12$ & $0 \cdot 26$ & $0 \cdot 18$ & $0 \cdot 26$ \\
\hline $4(24)$ & 0.39 & 0.81 & 0.58 & 0.92 & $0 \cdot 26$ & 0.33 & $0 \cdot 30$ & $0 \cdot 71$ & $0 \cdot 20$ & 0.40 \\
\hline $5(19)$ & $0 \cdot 12$ & $0 \cdot 29$ & $0-27$ & 0.64 & $0 \cdot 21$ & 0.36 & $0 \cdot 16$ & $0 \cdot 32$ & 0.05 & 0.09 \\
\hline $6(18)$ & 0.35 & 0.69 & $0 \cdot 18$ & $0 \cdot 49$ & $0 \cdot 13$ & $0 \cdot 24$ & $0 \cdot 12$ & 0.23 & $0 \cdot 16$ & 0.27 \\
\hline $7(18)$ & $0 \cdot 26$ & $0 \cdot 41$ & $0 \cdot 15$ & 0.35 & 0.04 & 0.11 & $0 \cdot 14$ & 0.22 & $0 \cdot 13$ & $0 \cdot 20$ \\
\hline $8(17)$ & $0 \cdot 12$ & $0 \cdot 22$ & $0 \cdot 11$ & 0.45 & 0.07 & 0.11 & 0.06 & $0 \cdot 18$ & 0.03 & $0 \cdot 16$ \\
\hline $9(18)$ & 0.13 & 0.41 & 0.49 & 1.02 & 0.02 & 0.08 & 0.13 & $0 \cdot 24$ & 0.13 & 0.24 \\
\hline $10(23)$ & 0.47 & 0.89 & 0.53 & 0.83 & $0 \cdot 14$ & 0.33 & $0 \cdot 13$ & $0 \cdot 33$ & $0 \cdot 15$ & 0.33 \\
\hline $11(21)$ & $0 \cdot 11$ & 0.23 & 0.85 & $1 \cdot 32$ & 0.06 & $0 \cdot 12$ & 0.06 & 0.14 & 0.08 & $0 \cdot 12$ \\
\hline \multicolumn{11}{|l|}{ R.I.C. } \\
\hline $1(27)$ & $0-51$ & 0.65 & 0.63 & 0.98 & 0.28 & 0.36 & $0 \cdot 16$ & 0.23 & 0.13 & 0.24 \\
\hline $2(23)$ & $0 \cdot 10$ & $0 \cdot 21$ & 0.33 & 0.75 & $0 \cdot 11$ & $0 \cdot 24$ & $0 \cdot 12$ & 0.23 & 0.04 & 0.13 \\
\hline $3(26)$ & $0 \cdot 27$ & $0-35$ & 0.61 & $1 \cdot 11$ & 0.09 & 0.12 & 0.19 & 0.24 & $0 \cdot 10$ & 0.15 \\
\hline $4(24)$ & 0.79 & 0.72 & $0 \cdot 71$ & 0.78 & 0.13 & 0.31 & 0.54 & 0.46 & 0.39 & 0.25 \\
\hline $5(19)$ & 0.05 & 0.39 & 0.12 & 0.27 & 0.11 & 0.24 & 0.08 & 0.19 & 0.05 & 0.20 \\
\hline $6(18)$ & 0.34 & 0.60 & 0.28 & 0.65 & 0.05 & $0 \cdot 11$ & 0.24 & 0.44 & $0 \cdot 20$ & 0.35 \\
\hline $7(\mathbf{1 8})$ & 0.17 & 0.37 & 0.20 & 0.51 & 0.02 & 0.13 & 0.05 & 0.17 & 0.06 & 0.12 \\
\hline $8(17)$ & 0.05 & $0 \cdot 11$ & 0.39 & 0.84 & 0.02 & $0 \cdot 10$ & 0.04 & 0.07 & 0.03 & 0.13 \\
\hline $9(18)$ & 0.07 & $0 \cdot 18$ & $0 \cdot 15$ & 1.68 & 0.03 & 0.07 & 0.08 & 0.18 & 0.07 & 0.13 \\
\hline $10(23)$ & $0 \cdot 32$ & 0.43 & 0.61 & 0.94 & 0.05 & 0.11 & 0.08 & $0 \cdot 16$ & 0.06 & $0 \cdot 13$ \\
\hline $11(21)$ & 0.17 & $0 \cdot 61$ & 0.36 & 0.66 & 0.09 & 0.18 & 0.08 & 0.16 & 0.03 & 0.08 \\
\hline
\end{tabular}

Values of 0.50 or less represent 'acceptable' representations of parent conversations. Values in parentheses in the first column represent the length in minutes of parent conversations. Upper half of table contains data for W.A.N.; the lower half contains data pertaining to R.I.C., the non-aphasic communication partner.

adequate in nearly all cases. For example, in table 3, conversation number 11 for N.K.Y. has a frequency of occurrence for self-repairs of 1.21 per minute. Comparing this with the values in table 7 , a 10 minute sample adequately represented the parent conversation $(\mathrm{CV}=0.29)$, but a 5 minute sample did not $(\mathrm{CV}=0.60)$. Using these criteria, there were 20 exceptions in 352 data points (excluding words per utterance, as they were not divided by the number of minutes), for a $94 \%$ ' hit' rate.

\section{Discussion}

This study examined the degree to which five variables were evenly distributed throughout conversations. With even distribution, smaller conversational segments could be used to estimate the occurrence in parent conversations. In examining conversation repair, for seven of the eight participants, 10 minute segments rarely 'missed the mark' for estimating self-repair frequency of parent conversations. The one exception, N.K.Y., rarely self-repaired, thus it was not surprising that these occurrences were unevenly distributed.

'Other-repair' usually took the form of requesting clarification from the other participant. If one were to use a 10 minute sample from the conversations in this study, those samples would yield a reasonable estimate of the parent conversation most of the time for six of the eight participants. Once again, N.K.Y. rarely requested clarification. Similarly, R.I.C. rarely used repair strategies more than twice per minute. 
Table 9. Coefficients of variation for dyad 3 (L.S.O. and S.Z.A.)

\begin{tabular}{|c|c|c|c|c|c|c|c|c|c|c|}
\hline \multirow{2}{*}{$\begin{array}{l}\text { Conversation } \\
\text { number }\end{array}$} & \multicolumn{2}{|c|}{ Self-repair/min } & \multicolumn{2}{|c|}{ Other-repair/min } & \multicolumn{2}{|c|}{ Words/utterance } & \multicolumn{2}{|c|}{ Words/min } & \multicolumn{2}{|c|}{ Utterances $/ \mathrm{min}$} \\
\hline & $10 \mathrm{~min}$ & $5 \mathrm{~min}$ & $10 \mathrm{~min}$ & $5 \mathrm{~min}$ & $10 \mathrm{~min}$ & $5 \mathrm{~min}$ & $10 \mathrm{~min}$ & $5 \mathrm{~min}$ & $10 \mathrm{~min}$ & $5 \mathrm{~min}$ \\
\hline \multicolumn{11}{|l|}{ L.S.O. } \\
\hline $1(20)$ & $0 \cdot 40$ & $0 \cdot 58$ & $0 \cdot 11$ & $0 \cdot 31$ & $0 \cdot 12$ & 0.19 & $0 \cdot 07$ & $0 \cdot 12$ & $0 \cdot 12$ & 0.18 \\
\hline $2(20)$ & 0.42 & $0 \cdot 86$ & 0.13 & $0 \cdot 25$ & 0.08 & $0 \cdot 18$ & 0.09 & 0.18 & 0.02 & 0.06 \\
\hline $3(18)$ & $0 \cdot 12$ & 0.35 & 0.23 & $0 \cdot 55$ & $0 \cdot 10$ & 0.22 & $0 \cdot 18$ & $0 \cdot 31$ & 0.09 & $0 \cdot 12$ \\
\hline $4(16)$ & 0.36 & 0.61 & 0.04 & $0 \cdot 38$ & 0.04 & $0 \cdot 14$ & 0.03 & $0 \cdot 11$ & 0.02 & 0.05 \\
\hline $5(16)$ & $0 \cdot 27$ & $0 \cdot 50$ & $0 \cdot 11$ & $0 \cdot 23$ & 0.06 & 0.15 & 0.06 & $0 \cdot 12$ & 0.02 & 0.04 \\
\hline $6(16)$ & 0.18 & 0.42 & $0 \cdot 19$ & $0 \cdot 41$ & 0.08 & 0.12 & 0.08 & $0 \cdot 22$ & 0.03 & 0.12 \\
\hline $7(17)$ & $0 \cdot 14$ & 0.55 & 0.22 & $0 \cdot 38$ & 0.13 & 0.21 & $0 \cdot 15$ & $0 \cdot 28$ & 0.05 & $0 \cdot 14$ \\
\hline $8(16)$ & 0.46 & $0 \cdot 35$ & $0 \cdot 10$ & 0.48 & 0.01 & 0.07 & 0.03 & 0.06 & 0.02 & 0.06 \\
\hline $9(16)$ & 0.11 & $0 \cdot 18$ & 0.39 & 0.80 & 0.09 & 0.18 & 0.06 & 0.08 & 0.06 & 0.15 \\
\hline $10(16)$ & $0 \cdot 16$ & 0.25 & 0.11 & 0.52 & 0.09 & $0 \cdot 20$ & 0.12 & 0.25 & $0-30$ & 0.39 \\
\hline $11(16)$ & 0.18 & $0 \cdot 40$ & 0.32 & 0.69 & 0.06 & 0.29 & 0.06 & 0.28 & 0.25 & 0.31 \\
\hline \multicolumn{11}{|l|}{ S.Z.A. } \\
\hline $1(20)$ & 0.14 & 0.35 & 0.17 & 0.47 & 0.04 & $0 \cdot 11$ & 0.09 & 0.18 & 0.06 & 0.12 \\
\hline $2(20)$ & 0.05 & $0 \cdot 16$ & 0.22 & 0.42 & 0.09 & 0.14 & 0.15 & 0.27 & 0.09 & 0.18 \\
\hline $3(18)$ & 0.11 & $0 \cdot 14$ & $0 . \overline{25}$ & 0.36 & 0.06 & 0.13 & 0.07 & $0 \cdot 13$ & 0.03 & 0.06 \\
\hline $4(16)$ & 0.13 & 0.25 & 0.14 & 0.33 & 0.01 & 0.07 & 0.11 & 0.11 & 0.03 & 0.06 \\
\hline $5(16)$ & 0.11 & 0.37 & 0.09 & 0.25 & 0.04 & 0.10 & 0.05 & $0 \cdot 16$ & 0.05 & 0.09 \\
\hline $6(16)$ & 0.13 & 0.31 & 0.12 & 0.27 & 0.05 & 0.14 & 0.10 & 0.19 & 0.07 & 0.10 \\
\hline $7(\mathbf{1 7})$ & $0-18$ & 0.36 & 0.25 & 0.42 & 0.07 & 0.15 & 0.05 & $0 \cdot 12$ & 0.03 & 0.05 \\
\hline $8(16)$ & 0.02 & $0 \cdot 27$ & 0.24 & 0.64 & 0.07 & 0.22 & 0.03 & $0 \cdot 14$ & 0.04 & 0.13 \\
\hline $9(16)$ & 0.26 & 0.52 & 0.11 & 0.25 & 0.08 & 0.09 & 0.11 & $0 \cdot 18$ & 0.05 & 0.13 \\
\hline $10(16)$ & 0.30 & 0.47 & 0.08 & 0.13 & $0 \cdot 10$ & 0.15 & 0.02 & 0.08 & 0.33 & 0.46 \\
\hline $11(16)$ & 0.22 & 0.42 & 0.13 & $0 \cdot 31$ & 0.05 & $0 \cdot 11$ & 0.06 & $0 \cdot 18$ & 0.13 & 0.33 \\
\hline
\end{tabular}

Values of 0.50 or less represent 'acceptable' representations of parent conversations. Values in parentheses in the first column represent the length in minutes of parent conversations. Upper half of table contains data for L.S.O.; the lower half contains data pertaining to S.Z.A., the non-aphasic communication partner.

Five minute conversations may be adequate for measuring speaking efficiency for some individuals. There were few exceptions in this study. Thus a clinician may estimate speaking efficiency from a reasonably brief sample.

This study brings conversational discourse analysis one step closer to clinical use. In short, behaviours which occur at least three times per minute can be estimated reliably with 5 minute samples. Behaviours which occur once per minute can be estimated reliably with 10 minute samples. With a minimum frequency of occurrence standard, clinicians can select variables likely to be amenable to CDA. Less frequently occurring variables can be monitored with larger sample sizes and/or using another method.

Two alternate solutions for using CDA to monitor less frequently occurring variables are to abandon the measurement of such variables or to develop a 'realtime' procedure. The former solution appears unsatisfactory, as many of the behaviours aphasiologists are concerned with may be unevenly distributed. However, it would be useful for the aphasiologist periodically to put aside the picture cards and workbooks and listen to a conversation for 10 minutes, monitoring such variables as repairs and speaking efficiency. A typical didactic therapy interaction might look like the following:

Client: I uh a dog.

Clinician: Yes, and what is this?

Client: Uh, pillow.

Clinician: Very good. 
Table 10. Coefficients of variation for dyad 4 (L.C.P. and C.H.S.)

\begin{tabular}{|c|c|c|c|c|c|c|c|c|c|c|}
\hline \multirow{2}{*}{$\begin{array}{l}\text { Conversation } \\
\text { number }\end{array}$} & \multicolumn{2}{|c|}{ Self-repair/min } & \multicolumn{2}{|c|}{ Other-repair/min } & \multicolumn{2}{|c|}{ Words/utterance } & \multicolumn{2}{|c|}{ Words/min } & \multicolumn{2}{|c|}{ Utterances/min } \\
\hline & $10 \mathrm{~min}$ & $5 \mathrm{~min}$ & $10 \mathrm{~min}$ & $5 \mathrm{~min}$ & $10 \mathrm{~min}$ & $5 \mathrm{~min}$ & $10 \mathrm{~min}$ & $5 \mathrm{~min}$ & $10 \mathrm{~min}$ & $5 \mathrm{~min}$ \\
\hline \multicolumn{11}{|l|}{ L.C.P. } \\
\hline $1(15)$ & $0 \cdot 06$ & $0 \cdot 15$ & $0 \cdot 31$ & 0.47 & 0.03 & $0 \cdot 14$ & $0 \cdot 04$ & 0.09 & 0.04 & 0.09 \\
\hline $2(15)$ & $0 \cdot 23$ & $0 \cdot 32$ & 0.34 & 0.62 & 0.07 & $0 \cdot 10$ & $0-11$ & 0.15 & 0.05 & 0.07 \\
\hline $3(15)$ & $0 \cdot 17$ & $0 \cdot 51$ & 0.23 & 0.58 & 0.07 & $0 \cdot 18$ & $0 \cdot 10$ & $0 \cdot 22$ & 0.04 & 0.05 \\
\hline $4(15)$ & 0.09 & 0.37 & $0 \cdot 25$ & 0.47 & 0.07 & 0.16 & 0.03 & $0 \cdot 18$ & $0 \cdot 04$ & 0.09 \\
\hline $5(15)$ & 0.07 & 0.20 & $0 \cdot 15$ & $6 \cdot 02$ & 0.05 & 0.15 & 0.05 & $0 \cdot 16$ & 0.05 & 0.09 \\
\hline $6(15)$ & 0.09 & 0.22 & 0.30 & 0.67 & 0.05 & $0 \cdot 17$ & 0.08 & 0.20 & 0.04 & 0.07 \\
\hline $7(15)$ & $0 \cdot 14$ & 0.32 & 0.01 & $0 \cdot 39$ & 0.04 & $0 \cdot 16$ & 0.08 & 0.20 & 0.06 & $0 \cdot 11$ \\
\hline $8(15)$ & $0 \cdot 10$ & 0.33 & 0.01 & $1 \cdot 25$ & 0.03 & 0.09 & 0.08 & $0 \cdot 18$ & 0.06 & 0.23 \\
\hline $9(15)$ & 0.35 & 0.59 & 0.53 & 0.49 & 0.11 & $0 \cdot 17$ & $0 \cdot 16$ & $0 \cdot 27$ & 0.06 & $0 \cdot 13$ \\
\hline $10(15)$ & 0.05 & 0.43 & 0.19 & 0.32 & 0.08 & $0 \cdot 16$ & 0.08 & $0 \cdot 15$ & 0.03 & $0 \cdot 10$ \\
\hline $11(15)$ & $0 \cdot 16$ & 0.33 & 0.14 & 0.30 & $0-11$ & 0.22 & $0 \cdot 14$ & $0 \cdot 31$ & 0.04 & $0 \cdot 14$ \\
\hline \multicolumn{11}{|l|}{$\begin{array}{l}11(15) \\
\text { C.H.S. }\end{array}$} \\
\hline $1(15)$ & 0.34 & 0.58 & 0.06 & 0.32 & 0.02 & 0.15 & 0.03 & $0 \cdot 16$ & 0.03 & $0 \cdot 10$ \\
\hline $2(15)$ & 0.28 & 0.60 & 0.05 & $0 \cdot 14$ & 0.07 & $0 \cdot 17$ & 0.07 & $0 \cdot 13$ & 0.03 & 0.09 \\
\hline $3(15)$ & 0.19 & 0.30 & $0 \cdot 15$ & 0.34 & 0.05 & 0.12 & 0.07 & 0.14 & 0.03 & 0.06 \\
\hline $4(15)$ & 0.15 & 0.36 & 0.17 & 0.28 & 0.05 & 0.22 & 0.08 & $0 \cdot 31$ & 0.05 & 0.15 \\
\hline $5(15)$ & 0.30 & 0.61 & 0.06 & 0.18 & 0.05 & 0.09 & $0 \cdot 15$ & 0.31 & 0.10 & 0.32 \\
\hline $6(15)$ & $0 \cdot 10$ & 0.44 & 0.05 & 0.29 & 0.03 & 0.12 & $0 \cdot 05$ & 0.27 & 0.05 & $0-20$ \\
\hline $7(15)$ & 0.24 & 0.45 & 0.09 & 0.27 & 0.04 & 0.09 & 0.09 & $0 \cdot 24$ & 0.06 & 0.17 \\
\hline $8(15)$ & 0.11 & $0 \cdot 25$ & $0 \cdot 13$ & 0.21 & 0.06 & $0 \cdot 17$ & 0.06 & $0 \cdot 19$ & 0.05 & $0 \cdot 17$ \\
\hline $9(15)$ & 0.51 & 0.72 & $0 \cdot 10$ & 0.45 & $0 \cdot 15$ & 0.25 & 0.29 & 0.37 & $0-14$ & 0.22 \\
\hline $10(15)$ & $0 \cdot 11$ & 0.48 & 0.24 & 0.60 & 0.04 & $0 \cdot 10$ & 0.10 & $0 \cdot 17$ & 0.06 & $0 \cdot 10$ \\
\hline $11(15)$ & 0.37 & 0.71 & 0.18 & $0 \cdot 36$ & 0.12 & 0.27 & 0.16 & 0.39 & 0.05 & 0.11 \\
\hline
\end{tabular}

Values of 0.50 or less represent 'acceptable' representations of parent conversations. Values in parentheses in the first column represent the length in minutes of parent conversations. Upper half of table contains data for L.C.P., while the lower half contains data pertaining to C.H.S., the nonaphasic communication partner.

A conversational interaction where repair strategies are included in the therapy objectives might look like the following:

Client: I uh a dog.

Spouse: The dog? What about the dog?

Client: Sick, sick.

Spouse: Oh yes, she's been sick since this morning.

Clinician: Very good. Reflecting 'What about the dog' seems to help.

The current study does not, of course, provide efficacy data for the use of such strategies. However, this study does support the reliable measurement of such data, given a minimum frequency of occurrence.

\section{References}

ARNEY, W. R. 1990, Understanding Statistics in the Social Sciences (NY, Freeman), pp. 73-74.

BESNIER, N. 1989, Information withholding as a manipulative and collusive strategy in Nukulaelae gossip. Language in Society, 18, 315-341.

BoLEs, L. 1996, When therapy works: conversation analysis on an individual who showed improvement in therapy. Unpublished paper presented at the Clinical Aphasiology Conference, Newport, Rhode Island.

Boles, L. 1997, Conversation analysis as a dependent measure in communication therapy with four individuals with aphasia. Asia Pacific Journal of Speech, Language and Hearing, 2, 43-61.

Brookshire, R. H. and Nicholas, L. S. 1994, Test-retest stability of measures of connected speech in aphasia. Clinical Apbasiology, 22, 119-133. 
Brookshire, R. H., Nicholas, L. S. and Krueger, K. 1978, Sampling of speech pathology treatment activities: an evaluation of momentary and interval sampling procedures. Journal of Speech and Hearing Research, 21, 652-667.

Damico, J. S. and Housewright, B. 1992, Conversational style-shifting and traumatic brain injury. A descriptive study. Unpublished paper presented at the American Speech-Language-Hearing Association Annual Convention, San Antonio.

Damico, J. S. and SCHweItzer, L. A. 1991, Getting the message: investigating transactional ability after mild head injury. Unpublished paper presented at the American Speech-LanguageHearing Association Annual Convention, Atlanta.

FERGuSON, A. 1994, The influence of aphasia, familiarity and activity on conversational repair. Apbasiology, 8, 143-157.

Fujiki, M., Brinton, B. and Sonnenber G, E. A. 1990, Repair of overlapping speech in conversations of specifically language-impaired and normally developing children. Applied Psycholinguistics, 11, 201-215.

Holland, A. H. 1980, Communicative Abilities in Daily Living (Baltimore: University Park).

Kennedy, M. R. T., Strand, E. A., Burton, W. and Peterson, C. 1994, Analysis of first-encounter conversations of right-hemisphere-damaged adults. Clinical Aphasiology, 22, 67-80.

Kertesz, A. 1982, The Western Aphasia Battery (NY: Grune \& Stratton).

McCormick, T. C. 1941, Elementary Social Statistics (NY: McGraw-Hill), p. 130.

PER KINs, L. 1995, Applying conversation analysis to aphasia : clinical implications and analytic issues. European Journal of disorders of Communication, 30, 372-383.

Psathas, G. 1995, Conversation Analysis: The Study of Talk-in-Interaction (Thousand Oaks, CA: Sage).

SACKs, H., SCHegloff, E. A., and JefFerson, G. 1974, A simplest systematics for the organization of turn-taking for conversation. Language, 50, 696-735.

Simmons-Mackie, N. N. and Damico, J. 1996, Accounting for handicaps in aphasia: communicative assessment from an authentic social perspective. Disability and Rebabilitation, 18, 540-549.

Tomasello, M., Conti-Ramsden, G. and Ewert, B. 1990, Young children's conversations with their mothers and fathers: differences in breakdown and repair. Journal of Cbild Language, 17, 115-130.

WeIngarten, K. 1992, A consideration of intimate and non-intimate interactions in therapy. Family Process, 31, 45-59. 